\title{
Theoretical considerations in regard to the demonstration of musculoskeletal and cardiovascular interactions
}

FELIX J. ROGERS, D.O.

Trenton, Michigan

A central tenet of original osteopathic philosophy and theory is that disturbances in visceral function can produce altered musculoskeletal function. Likewise, abnormalities of the musculoskeletal system are considered to be a potential cause of some form of visceral disease. A review $^{1}$ of the osteopathic literature on viscerosomatic reflexes, which summarized findings of musculoskeletal abnormalities in a wide variety of visceral disturbances, was published recently. There have been no studies demonstrating that heart disease may be caused by reflex-mediated musculoskeletal abnormalities. More interest has been generated by attempts to show that osteopathic manipulative treatment (OMT) affects cardiac function. Thus, the literature to date reflects considerable observations of viscerosomatic reflex changes and virtually no evidence of somatovisceral interactions.

Recent studies have shown palpatory musculoskeletal alterations in the upper part of the thoracic spine of patients with chronic coronary artery disease $(\mathrm{CAD})^{2,3}$ and acute myocardial infarction. ${ }^{4}$ It is not clear whether the slight variation in the thoracic segments involved reflects sample size, the nature of underlying heart disease, a different definition of the musculoskeletal lesion, or biologic variability. The large number of descriptors employed by Nicholas and coauthors ${ }^{4}$ for recording musculoskeletal findings versus the simple terms used by Cox and associates ${ }^{3}$ highlights the need for standard terminology.

Nearly all studies of the musculoskeletal component in heart disease have involved patients with chronic $\mathrm{CAD}$ or acute myocardial infarction, probably because of their high prevalence and the wellestablished methods available to diagnose these visceral diseases. Should we expect all cardiovascular diseases to show the same musculoskeletal manifestations as $\mathrm{CAD}$ ? A review of the situation suggests that some cardiac states may have a higher penetrance into the musculoskeletal system than others. This report will review anatomic and physiologic considerations that may be relevant to the kinds of possible interactions between the cardiovascular and musculoskeletal systems. In addition, suggestions will be offered to assess the effects of OMT on cardiovascular function.

\section{The autonomic nervous system in cardiovascular-musculoskeletal interactions}

Classically, a stimulus-response sequence must involve the following: (1) a receptor; (2) an afferent pathway; (3) an efferent pathway; and (4) an effector end organ. A viscerosomatic lesion may occur when visceral afferent nerves interconnect with cell bodies of somatic efferent nerves at segmental levels of the spinal cord to produce a local reaction in the musculoskeletal system, which is characterized by abnormalities of range of motion, soft tissue texture, et cetera. Interest has developed in the possibility of reflexes from the heart that have the afferent nerve entering the spinal cord directly through sympathetic nerves, ${ }^{5}$ especially because this may provide better understanding of the cardiac viscerosomatic response.

Evidence for the role of cardiogenic reflexes in a number of clinical situations is growing. ${ }^{6,7}$ Some afferent nerve fibers travel with sympathetic nerves and may be chemosensitive, mechanosensitive, or both. ${ }^{8}$ Sympathetic afferents apparently mediate the sensation of cardiac pain..$^{9-11}$ Other afferent nerves travel with the vagus ${ }^{12}$; they are 
known to mediate depressor responses produced by ischemia or stretch of left ventricular myocar-

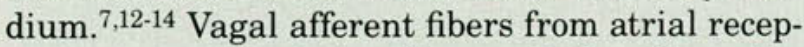
tors may participate in reflex regulation of vascular volume. ${ }^{15}$ The afferent cardiac vagal nerve fibers travel to the medulla. The afferent cardiac sympathetic nerve fibers, however, run to the fifth and sixth thoracic segments of the spinal cord. ${ }^{16}$

In theory, for cardiogenic reflexes to involve reflection into the segmentally related musculoskeletal system, the afferent limb should involve the sympathetic nervous system. One might anticipate that disease states characterized by cardiogenic reflexes traveling by way of vagal afferents would not show the same musculoskeletal response seen with sympathetic afferent nerves. Examples of cardiac conditions associated with vagally mediated reflexes include atrial fibrillation and paroxysmal supraventricular tachycardia (via left atrial stretch receptors), ${ }^{15,17}$ acute inferior myocardial infarction, and ischemia (Bezold-Jarisch reflex $)^{7}$ and early heart failure (left and right atrial receptors). ${ }^{18}$ (Chronic heart failure represents a complex entity with depleted cardiac receptors, ${ }^{19}$ attenuated reflex responses, and abnormal sympathetic and parasympathetic nervous system function, and will be referred to subsequently.) The musculoskeletal system has not been evaluated by palpatory examination in any of these conditions as separate clinical entities.

A musculoskeletal component to coronary artery disease has been demonstrable, presumably because angina pectoris is mediated via sympathetic nerves that enter the spinal cord. Should we expect a similar finding in other types of heart disease? It is possible that cardiac conditions associated with sympathetic afferents may have a greater penetration into the segmental musculoskeletal system than conditions associated with parasympathetic afferent stimulation. In addition, it is conceivable that some responses may be mixed. For example, patients with aortic stenosis may present with syncope, which is thought to be due to ventricular baroreceptor-mediated hypervagotonia, ${ }^{20,21}$ whereas angina in the same condition may be mediated via sympathetic fibers. Patients with syncope as the primary symptom of aortic stenosis may have few or no concomitant musculoskeletal changes, whereas those with angina may have findings like those in patients with coronary artery disease; the musculoskeletal response will therefore be dependent upon the patient's clinical presentation. In a similar fashion, it has been reported that inferior myocardial ischemia and infarction are associated with vagally mediated vasodepressor response, whereas anterior myocardial infarction is associated with increased sympathetic nervous system tone. ${ }^{22}$ Therefore, musculoskeletal findings may be different in patients with inferior versus anterior infarction or ischemia. Another consideration involves mitral valve prolapse, in which increased adrenergic tone has been demonstrated ${ }^{23}$ Because the source of this adrenergic hyperactivity may be of neuroendocrine origin, rather than cardiogenic, there may not be a specific, segmentally related somatic component.

The somatic expression of a variety of well-defined cardiac conditions has not been evaluated systematically. Such an evaluation could provide valuable insights into the nature of viscerosomatic interactions, and might also add further to the knowledge of cardiogenic reflexes. Additional information in regard to the time course of reflex-mediated somatic dysfunction might come from analysis of patients subjected to percutaneous transluminal coronary angioplasty (PTCA), an invasive procedure that affects coronary artery occlusive disease with less musculoskeletal perturbation than coronary artery bypass graft surgery. For example do palpatory musculoskeletal lesions disappear after successful PTCA? If so, over what time period? If coronary restenosis occurs, is it before or after musculoskeletal changes occur? Is there any association between the angiographic distribution of the lesions and the musculoskeletal findings, especially with inferior versus anterior locations?

\section{Assessing effects of OMT on cardiovascular function}

If there is a viscerosomatic interaction, one must ask why studies have failed to demonstrate a change in cardiac function in response to OMT. One might presume that the effect was too small to be detected by the measurements used, or that the time course of response was not assessed properly, or that the effect was a response to repetitive and not single manipulative treatment, or that an inappropriate clinical model was selected. Another consideration is more delicate: Did the OMT achieve its goals in normalizing musculoskeletal function? If there are abnormalities of range of motion or soft tissue texture and OMT does not restore normalcy, should that intervention represent a valid test of OMT? By analogy, tests of vasodilating agents for the treatment of congestive heart failure require a certain drop in blood pressure to ensure an adequate drug dose. It seems reasonable that the response to OMT should be assessed independently during clinical trials. It is unfortunate that virtually all studies with OMT have failed to include this evaluation.

It is now generally thought that any effects of 
OMT on the visceral system would be mediated via the autonomic nervous system (ANS). This relationship is most clear in terms of inhibition of a reflex mediated primarily by the sympathetic nervous system, but the possibility of a modulating influence on parasympathetic stimulation should also be considered. As such, an effect of OMT should be most clearly demonstrated in those situations where the heart disease is caused by, or associated with, abnormalities of the ANS (Table 1). Furthermore, if the efficacy of OMT involves the normalization of abnormal somatovisceral reflexes, one would expect to demonstrate little change in baseline hemodynamic values if they are already normal prior to treatment. In this situation, one might demonstrate alterations in measures of autonomic function (Table 2) in response to OMT in addition to, or instead of, changes in standard hemodynamic measurements, such as cardiac output or left ventricular filling pressure. The possibility of a confounding influence of cortical and/or central modulation of ANS function needs to be considered, and trials should be designed to include this possibility.

For example, congestive heart failure represents a clinical situation associated with deranged sympathetic and parasympathetic nervous system control. ${ }^{24,25}$ There is increased adrenergic tone associated with disappearance of sinus arrhythmia, abnormal heart rate and blood pressure responses to Valsalva maneuver and tilting, and elevated serum norepinephrine levels. Several of these responses have been suggested as diagnostic methods for the detection of heart disease. ${ }^{26}$ With the patients serving as their own controls, these parameters may be assessed before and after OMT. In addition, systolic time intervals in this circumstance might indicate if any changes in myocardial performance occurred in response to alterations of the ANS.

A pilot study ${ }^{27}$ of 10 patients with CHF demonstrated orthostatic hypotension in 8 patients in the control state but in only 3 patients following OMT. Resting heart rate, blood pressure, and systolic time intervals did not change in a significant fashion following OMT in this small group of patients.

The potential value of OMT in patients with heart failure is unclear and unexplored. It is a possibility that the hyperadrenergic state contributes to the pathophysiology of this entity. Before manipulation is recommended as an adjunct to therapy for patients with heart failure, it would be useful to know the effect of such treatment on the ANS.

\section{Comment}

Cardiovascular abnormalities represent a hetero-

\begin{tabular}{l} 
TABLE 1. CARDIAC CONDITIONS ASSOCIATED WITH CLINICALLY \\
IMPORTANT ALTERATIONS IN ANS FUNCTION. SOME CONDI- \\
TIONS, SUCH AS HEART FAILURE, ARE CONSISTENTLY ASSOCI- \\
ATED WITH ANS ABNORMALITIES; OTHERS INVOLVE TRAN- \\
SIENT ALTERATIONS THAT MAY BE ASSOCIATED WITH AN \\
ACUTE CARDIAC EVENT. \\
\hline 1. Heart failure \\
2. Coronary artery disease \\
3. Coronary vasospasm \\
4. Mitral valve prolapse \\
5. Supraventricular tachycardia \\
6. Ventricular tachycardia \\
7. Sudden cardiac death \\
8. Bradycardia
\end{tabular}

TABLE 2. SELECTED TESTS OF AUTONOMIC FUNCTION (MODIFIED FROM BANNISTER, ${ }^{28}$ WITH PERMISSION).

1. Sympathetic efferent constrictor fibers to capacity and resistance vessels:
(a) Postural hypotension
(b) Lack of blood pressure "overshoot" after Valsalva ma- neuver
(c) Lack of blood pressure rise on stress
(d) Low resting plasma noradrenalin
(e) Lack of rise of plasma noradrenalin on tilting

2. Sympathetic efferent fibers to heart:
(a) Lack of tachycardia during Valsalva maneuvers, phase II
(b) Lack of tachycardia during tilting
(c) Lack of tachycardia on cortical arousal
(d) Lack of tachycardia on isometric exercise

3. Parasympathetic efferent fibers to heart:
(a) Lack of sinus arrhythmia
(b) Lack of effect of carotid massage
(c) Lack of rise of cardiac rate with atropine

geneous array of disease states. A variety of conditions are associated with clinically important cardiogenic reflexes. Those mediated by sympathetic afferent nerves are more likely to be associated with segmental somatic dysfunction than those mediated by vagal afferent nerves. The possibility of variable expression of musculoskeletal markers of different cardiac diseases should be investigated systematically. There presently appear to be data supporting the existence of a musculoskeletal component to coronary artery disease.

A clinical model for the investigation of the effects of osteopathic manipulative treatment on cardiac disease should involve a disease state associated with abnormal function of the ANS. It is suggested that measures of autonomic function be evaluated in addition to other, standard hemodynamic variables. It may be that evidence of an ANS-mediated interaction between the musculoskeletal and cardiovascular systems should be sought before efforts are made to test the efficacy of OMT for cardiovascular disease. 
1. Beal, M.C.: Viscerosomatic reflexes. A review. JAOA 85:786-801, Dec 85 2. Beal, M.C.: Palpatory testing for somatic dysfunction in patients with cardiovascular disease. JAOA 82:822-31, Jul 83

3. Cox, J.M., et al.: Palpable musculoskeletal findings in coronary artery disease. Results of a double-blind study. JAOA 82:831-6, Jul 83

4. Nicholas, A.S., et al.: A somatic component to myocardial infarction. Br Med J 291:13-7, Jul 85

5. Malliani, A., et al.: Spinal sympathetic cardiocardiac reflexes. Circ Res 30:158-66, Feb 72

6. Linden, R.J.: Reflexes from the heart. Progr Cardiovasc Dis 18:201-21, Nov-Dec 75

7. Mark, A.L.: The Bezold-Jarisch reflex revisited. Clinical implications of inhibitory reflexes originating in the heart. J Am Coll Cardiol 1:90-102, Jan 83

8. Coleridge, J.C.G., and Coleridge, H.M.: Chemoreflex regulation of the heart. In The handbook of physiology, section 2: The cardiovascular system, vol. 1, The heart, edited by R.M. Berne. American Physiological Society, Bethesda, Md., 1979

9. Harken, D.E., et al.: De-epicardialization. A simple, effective surgical treatment for angina pectoris. Circulation 12:955-62, Dec 55

10. Lindgren, I., and Olivecrona, H.: Surgical treatment of angina pectoris. J Neurosurg 4:19-39, Jan 47

11. Foreman, R.D., Ohata, C.A., and Gerhart, K.D.: Neural mechanism underlying cardiac pain. In Neural mechanisms in cardiac arrhythmias (Perspectives in cardiovascular research, vol. 2), edited by P.J. Schwartz, et al. Raven Press, New York, 1978

12. Sleight, P.: A cardiovascular depressor reflex from the epicardium of the left ventricle in the dog. J Physiol 173:321-43, Sep 64

13. Coleridge, H.M., Coleridge, J.C.G., and Kidd, C.: Cardiac receptors in the dog, with particular reference to two types of afferent ending in the ventricular wall. J Physiol 174:323-39, Nov 64

14. Paintal, A.S.: Vagal sensory receptors and their reflex effects. Physiol Rev 53:159-227, Jan 73

15. Zucker, I.H., and Gilmore, J.P.: Atrial receptor modulation of renal function in heart failure. In Disturbances in neurogenic control of the circulation, edited by F.M. Abboud, et al. American Physiological Society, Bethesda, Md., 1981

16. Brown, A.M.: Cardiac reflexes. In op. cit., ref. 8

17. Wood, P.: Polyuria in paroxysmal tachycardia and paroxysmal atrial flutter and fibrillation. Br Heart J 25:273-82, 1963
18. Abboud, F.M., Thames, M.D., and Mark, A.L.: Role of cardiac afferent nerves in regulation of circulation during coronary occlusion and heart failure. In op. cit., ref. 15

19. Bristow, M.R., et al.: Decreased catecholamine sensitivity and $\beta$ adrenergic-receptor density in failing human hearts. N Engl J Med 307:205-11, 22 Jul 82

20. Sleight, P.: Cardiac vomiting. Br Heart J 46:5-7, Jul 81

21. Richards, A.M., et al.: Syncope in aortic valvular stenosis. Lancet 2:1113-7, Nov 84

22. Pantridge, J.F.: Autonomic disturbance at the onset of acute myocardial infarction. In op. cit., ref. 11

23. Boudoulas, H., et al.: Metabolic studies in mitral valve prolapse syndrome. A neuroendocrine-cardiovascular process. Circulation 61:1200-5, Jun 80

24. Braunwald, E., et al.: Congestive heart failure. Biochemical and physiological considerations. Combined clinical staff conference at the National Institutes of Health. Ann Intern Med 64:904-41, Apr 66

25. Eckberg, D.L., Drabinsky, M., and Braunwald, E.: Defective cardiac parasympathetic control in patients with heart disease. N Engl J Med $285: 877-83,14$ Oct 71

26. Greenberg, P.S., et al.: Value of autonomic maneuvers in detecting cardiac disease. Pract Cardiol 9:88-100, Oct 83

27. Rogers, F., Glassman, J., and Kavieff, R.: Effects of osteopathic manipulative treatment on autonomic nervous system function in patients with congestive heart failure (abstract). JAOA 86:605, Sep 86

28. Bannister, R., ed.: Autonomic failure. A textbook of clinical disorders of the autonomic nervous system. Oxford University Press, New York, 1983

\section{Accepted for publication in March 1986.}

Dr. Rogers is an associate clinical professor of internal medicine at Michigan State University-College of Osteopathic Medicine. $\mathrm{He}$ is in the private practice of cardiology at Riverside Osteopathic Hospital and Detroit Osteopathic Hospital.

Dr. Rogers, 2205 Riverside Drive, Trenton, Michigan 48183. 
ive your angina patients hat they're missing...

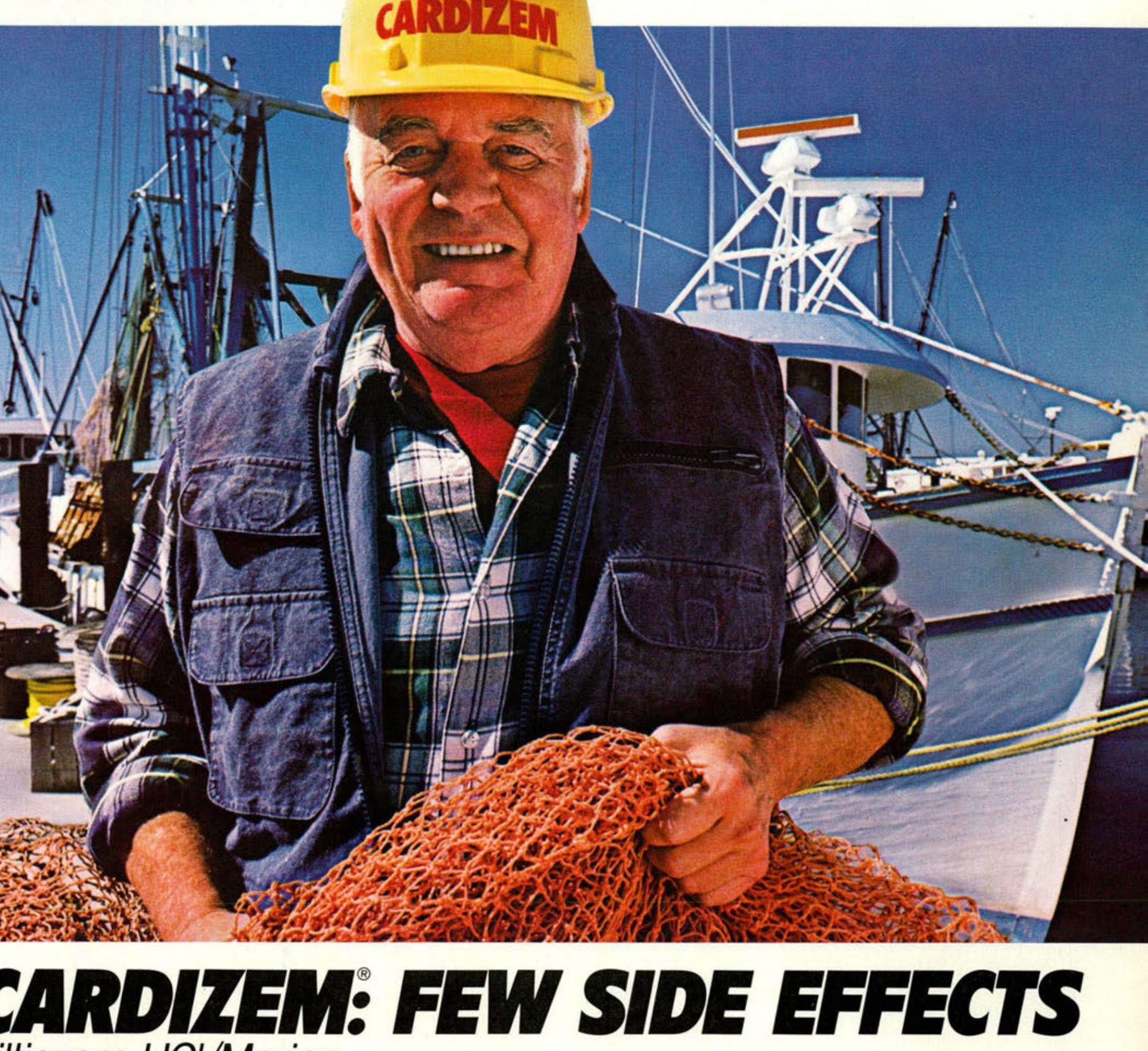

Itiazem $\mathrm{HCl} /$ Marion

Antianginal action includes dilatation of coronary arteries, a decrease in vascular resistance/afterload, and a reduction in heart rate

Proven efficacy when used alone in angina'

Compatible with other antianginals ${ }^{2,3}$

A safe choice for angina patients with coexisting hypertension, asthma, COPD, or PVD ${ }^{4,5}$

*See Warnings and Precautions. 


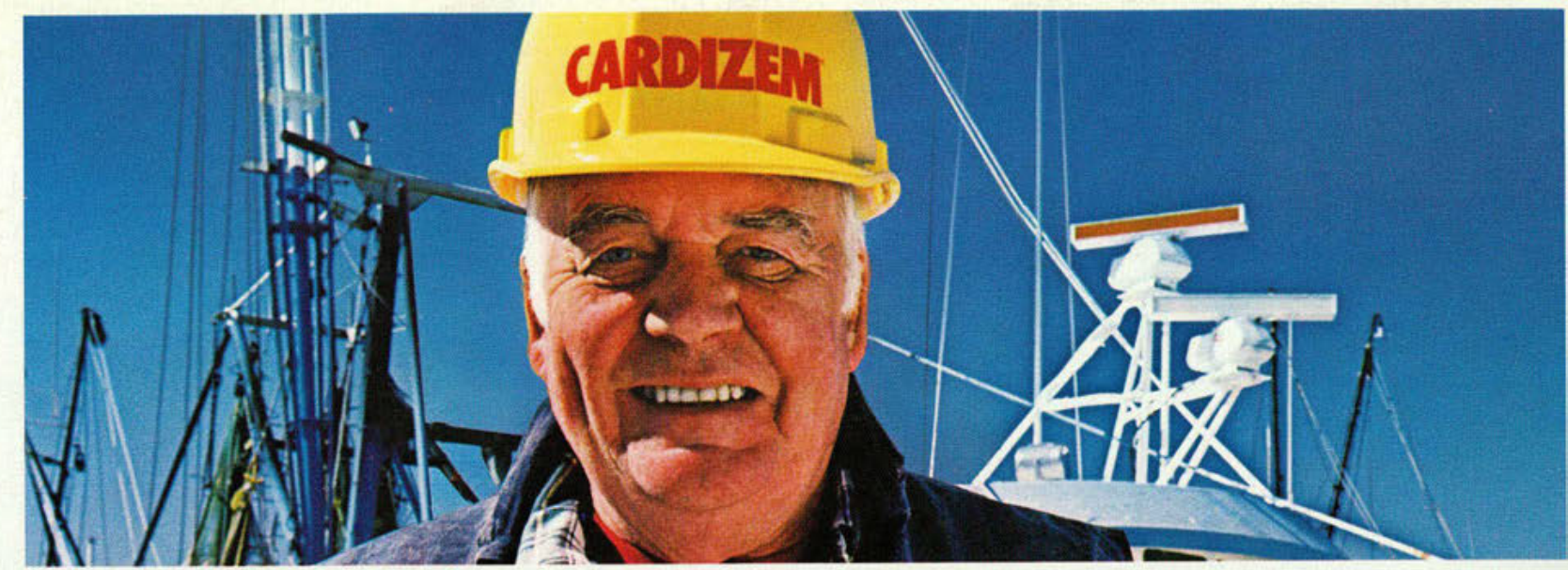

\section{CARDHZEM FEW SIDE EFFECTS diltiazem HCI/Marion IN ANTIANGINAL THERAPY}

\section{$60 \mathrm{mg}$ tid or qid}

Brief Summary

Professional Use Information

\section{CARDIZEM}

(dilfiazem $\mathrm{HCl}$ ) $30 \mathrm{mg}$ and $60 \mathrm{mg}$ Tablets

\section{CONTRAINDICATIONS}

CARDIZEM is contraindicated in (1) patients with sick sinus syndrome except in the presence of a functioning ventricular pacemaker, (2) patients with second- or third-degree AV block except in the presence of a functioning ventricular pocemaker, and (3) patients with hypotension (less than $90 \mathrm{~mm} \mathrm{Hg}$ systolic).

\section{WARNINGS}

1. Cardiac Conduction. CARDIZEM prolongs AV node refractory periods without significantly prolonging sinus node recovery time, except in patients with sick sinus syndrome. This effect may rarely result in abnormally slow heart rates (particularly in patients with sick sinus syndrome) or second- or third-degree AV block (six of 1,243 patients for $0.48 \%$ ). Concomitant use of diltiazem with beta-blockers or digitalis may result in additive effects on cardiac conduction. A patient with Prinzmetal's angina developed periods of asystole (2 to 5 seconds) after a single dose of $60 \mathrm{mg}$ of diltiazem.

2. Congestive Heart Failure. Although diltiazem has a negative inotropic effect in isolated animal tissue preparations, hemodynamic studies in humans with normal ventricular function have not shown a reduction in cardiac index nor consistent negative effects on contractility (dp/dt).

Experience with the use of CARDIZEM

alone or in combination with beta-blockers in potients with impaired ventricular function is very limited. Caution should be exercised when using the drug in such patients.

3. Hypotension. Decreases in blood pressure associated with CARDIZEM therapy may occasionally result in symptomatic hypotension.

4. Acute Hepatic Injury, In rare instances, significant elevations in enzymes such as alkaline phosphatase, CPK, LDH, SGOT, SGPT, and other symptoms consistent with acute hepatic injury hove been noted. These reactions have iveen reversible upon discontinuation of drug therapy. The relationship to CARDIZEM is uncertain in most cases, but probable in some. (SEe PRECAUTIONS.)

\section{PRECAUTIONS}

General. CARDIZEM (diltiazem hydrochloride) is extensively metabolized by the liver and excreted by the kidneys and in bile. As with any new drug given over prolonged periads, laboratory parameters should be monitored at regular intervals. The drug should be used with caution in patients with impaired renal or hepatic function. In subacute and chronic dog and rat studies designed to produce toxicity high doses of diltiazem were associated with hepatic damage. In special subacute hepatic studies, oral doses of $125 \mathrm{mg} / \mathrm{kg}$ and higher in rats were associated with histological changes in the liver which were reversible when the drug was discontinued. In dogs, doses of $20 \mathrm{mg} / \mathrm{kg}$ were also associated with hepatic changes; however, these changes were reversible with continued dosing.

Drug Interaction. Pharmacologic studies indicate that there may be additive effects in prolonging AV conduction when using beta-blackers or digitalis concomitantly with CARDIZEM (See WARNINGS)

Controlled and uncontrolled domestic studies suggest that concomitant use of CARDIZEM and beta-blockers or digitalis is usually well tolerated. Available data are not sufficient, however, to predict the effects of concomitant treatment, particularly in patients with left ventricular dysfunction or cardiac conduction abnormalities. In healthy volunteers, dilfiazem has been shown to increase serum digoxin levels up to $20 \%$

Carcinogenesis, Mutagenesis, Impaiment of Fertility. A 24-month study in rats and a 21 -month study in mice showed no evidence of carcinogenicity. There was also no mutogenic response in in vitro bacterial tests. No intrinsic effect on fertility was observed in rots.

Pregnancy. Category C. Reproduction studies have been conducted in mice, rats, and rabbits. Administration of doses ranging from five to ten times greater (on a ma/kg basis) than the daily recommended therapeutic dose has resulted in embryo and fetal lethality. These doses, in some studies, have been reported to cause skeletal abnormalifies. In the perinatal/postnatal studies, there was some reduction in early individual pup weights and survival rates. There was an increased incidence of stillbirths at doses of 20 times the human dose or greater.

There are no well-controlled studies in pregnant women; therefore, use CARDIZEM in pregnant women only if the potential benefit justifies the potential risk to the fetus

Nursing Mothers. Diltiazem is excreted in human milk. One report suggests that concentrations in breast milk may approximate serum levels. If use of CARDIZEM is deemed essential, an alternative method of infant feeding should be instituted

Pediatric Use. Safety and effectiveness in children have not been established.

\section{ADVERSE REACTIONS}

Serious adverse reactions have been rare in studies carried out to date, but it should be recognized that patients with impaired ventricular function and cardiac conduction abnormalities have usually been excluded.

In domestic placebo-controlled trials, the incidence of adverse reactions reported during CARDIZEM theropy was not greater than that reported during placebo therapy.

The following represent accurrences observed in clinical studies which can be at least reasonably asso- ciated with the pharmacology of calcium influx inhibition In many cases, the relationship to CARDIZEM has not been established. The most common occurrences as we as their frequency of presentation are: edema (2.4\%), headache $(2.1 \%)$, nausea $(1.9 \%)$, dizziness $(1.5 \%)$, rash (1.3\%), asthenia (1.2\%). In addition, the following events were reported infrequently (less than $1 \%$ ):

Cardiovascular: Angina, ambythmia, AV block (first degree), AV block (second or thir degree - see conduction warning bradycardia, congestive heart failure, flushing, hypotension, palp fations, syncope.

Nervous System: Amnesia, gait abnormality halluci nations, insomnia, nervousness, paresthesia, personality change, somnolence, tinnitus, tremor.

Gostrointestinal: Anorexia, constipation, diamhea dysgeusia, dyspepsia, mild elevations of alkaline phosphatose SGOT SGPT and LDH (see hepotic warnings), vomiting weight increase.

Dermatologic: Petechiae, pruritus, photosensitivit Other: unticario

Amblyopia dyspnea, epistoxis, ey imitation, hyperglycemia, nasal congestion, nocturia, ostecarticulo pain, polyuria, sexual difficulties

The following postmarketing events have been reported infrequently in potients receiving CARDIZEM: alopecia, gingival hyperplasia, erythema mulfiforme, a leukopenia. However, a definitive cause and effect between these events and CARDIZEM therapy is yet to be established. Issued See complete Professional Use Information before prescribing.

References: 1. Pepine WJ, Feldman RL, Hill JA et al: Clinical outcome after treatment of rest angina with calcium blockers: Comparative experience during the initial year of therapy with diltiazem, nifedipine, and verapamil. Am Heart J 1983; 106(6): 1341-1347 2. Shapiro W: Calcium channel blockers: Actions on th heart and uses in ischemic heart disease. Consultant 1984 24(Dec): 150-159. 3. Johnston DL, Lesoway R, Humen DP et al: Clinical and hemodynamic evaluation propranolol in combination with verapamil, nifedipine and dilfiazem in exertional angina pectoris: A placebocontrolled, double-blind, randomized, crossover study Am J Cardiol 1985;55:680-687. 4. Cohn PF, Braunw E. Chronic ischemic heart disegse in Braunwald E (ed Heart Disease: A Textbook of Cardiovascular Medicine ed 2. Philadelphia, WB Saunders Co, 1984, chop 39 5. Schroeder JS: Calcium and beta blockers in ischem heart disease: When to use which. Mod Med

1982,50(Sept):94-116. 